\title{
Unveiling Excited-State Chirality of Binaphthols by Femtosecond Circular Dichroism and Quantum-Chemical Calculations
}

Marco Schmid ${ }^{\dagger}$, Lara Martinez-Fernandez ${ }^{\S, l}$, Dimitra Markovitsi ${ }^{\S}$, Fabrizio Santoro $\$$, François Hache ${ }^{\dagger}$, Roberto Improta $a^{\S \neq}$, Pascale Changenet ${ }^{\dagger *}$

${ }^{\dagger}$ Laboratoire d'Optique et Biosciences, CNRS, INSERM, Ecole polytechnique, Institut Polytechnique de Paris, 91128 Palaiseau cedex, France

${ }^{\S}$ LIDYL, CEA, CNRS, Université Paris-Saclay, F-91191 Gif-sur-Yvette, France

'Departamento de Química, Facultad de Ciencias, Modúlo13, Universidad Autónoma de Madrid, Campus de Excelencia UAM-CSIC, Cantoblanco, 28049 Madrid, Spain

${ }^{\$}$ Consiglio Nazionale delle Ricerche, Istituto di Chimica dei Composti Organometallici, SS di Pisa, Area della Ricerca, via G. Moruzzi 1, I-56124 Pisa, Italy

${ }^{\ddagger}$ Istituto Biostrutture e Bioimmagini, Consiglio Nazionale delle Ricerche, Via Mezzocannone 16, I-80134 Napoli, Italy

\section{SUPPORTING INFORMATION}

\section{Table of contents}

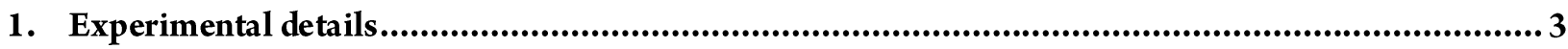

1.1. Sample preparation and steady-state measurements.........................................................................................

1.2. Time-resolved absorption and $\mathrm{CD}$ measurements............................................................................................

2. Quantum chemical calculations .........................................................................................................4

2.1. Computational details .........................................................................................................................................

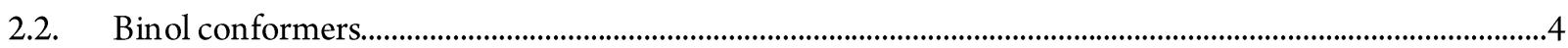

2.3. Electronic excitations of $\left(\mathrm{S}_{\mathrm{a}}\right)$-binol, $\left(\mathrm{R}_{\mathrm{a}}\right)-\mathrm{PL1},\left(\mathrm{R}_{\mathrm{a}}\right)-\mathrm{PL2}$ and 2-naphthol....................................................5

2.4. Electronic excitations of $\left(S_{a}\right)$-binol obtained by using the $M 11$ functional....................................................6

2.5. Detailed analysis of the torsional potential energy surfaces ..............................................................................

2.6. Computed static $\mathrm{CD}$ spectra of binols at the Boltzman equilibrium...................................................................

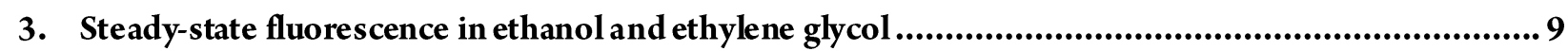

4. Additional time-resolved absorption and CD measurements....................................................... 10

4.1. Test for the validity of the TR-CD set-up......................................................................................................... 10

4.2. Achiral transient absorption measurements ......................................................................................................... 10

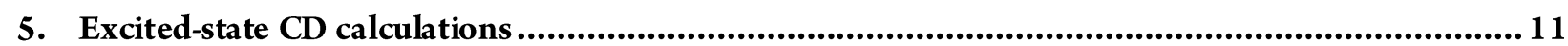

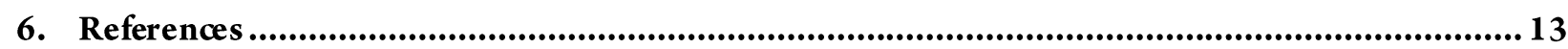




\section{Experimental details}

\subsection{Sample preparation and steady-state measurements}

$\left(S_{a}\right)$ - and $\left(R_{a}\right)$-binols were purchased from Sigma-Aldrich with a purity of $>97 \%$. The bridged compounds, $\left(R_{a}\right)$-PL1 and $\left(R_{a}\right)$-PL2, were synthesized as previously described. ${ }^{1}$ All solutions were sonicated before use. Static absorption and CD spectra were recorded with a double-beam Cary 100 (Agilent) spectrophotometer and a homemade spectropolarimeter, respectively. Static fluorescence spectra were measured with a spectrofluorimeter SPEX Fluorolog 3 (Jobin Yvon). The fluorescence spectra were corrected from the wavelength dependent response of the instrument. Typically, time-resolved experiments were performed with a sample concentrations of $\sim 2.3 \times 10^{-4} \mathrm{~mol}^{-\mathrm{L}^{-1}}$ and static CD measurements with sample concentrations of $\sim 1 \times 10^{-4} \mathrm{~mol} . \mathrm{L}^{-1}$.

\subsection{Time-resolved absorption and CD measurements}

Time-resolved experiments were carried out with a pump-probe set-up using an amplified $1 \mathrm{kHz}$ Ti:sapphire laser system (Spectra-Physics, Solstice) delivering 100-fs pulses at $800 \mathrm{~nm}$ with an energy of $3 \mathrm{~mJ}$. The pump pulses at $266 \mathrm{~nm}$ were obtained from the third harmonic generation of the fundamental pulses at $800 \mathrm{~nm}$ and focused onto a $\sim 0.4 \mathrm{~mm}^{2}$ surface in a 1-mm optical-path length flow silica cell. The pump energy was set to $0.5 \mu \mathrm{J}\left(\sim 125 \mathrm{~mW} . \mathrm{cm}^{-2}\right)$ to avoid photo-degradation of the studied compounds and the sample cell windows during measurements. Probe pulses at $234 \mathrm{~nm}$ with an energy of $150 \mathrm{~nJ}$ were generated by parametric amplification of white light continuum followed by the sum of frequency generation with the second harmonic of the $800-\mathrm{nm}$ laser pulses. The UV probe beam was attenuated and focused in the sample cell with a typical diameter of $100 \mu \mathrm{m}$. The transmitted probe beam was collected by a photomultiplier tube connected to a boxcar integrator.

TR-CD experiments were performed by measuring the variation of the probe beam ellipticity with the combination of an uncoated Babinet-Soleil (BS) compensator and two crossed polarizers (Glan-Thompson $\alpha$-BBO, extinction ratio 105:1), as previously described. ${ }^{2}$ The probe beam intensity transmitted by the sample was alternatively measured with and without the pump modulated by a chopper at $250 \mathrm{~Hz}$. Signals were typically averaged over 1000 laser shots. The pump and probe polarization was set to a parallel orientation with a common polarizer to avoid contribution from the sample birefringence to the differential $C D$ signals $(\triangle C D)$. For each pump-probe delay, the probe intensity was measured for 20 different $B S$ retardations. Under these conditions, acquisition of $\Delta C D$ kinetics traces with a precision of ca. \pm 5 mdeg required about $5 \mathrm{~h}$. The output probe intensity as a function of the BS retardation is a parabola. The shift and the curvature changes of the parabola measured alternatively with and without the pump, yield directly the pump-induced $C D(\triangle C D)$ and transient absorption changes $(\Delta \mathrm{A})$, respectively. In the following, we note $C D=A_{L^{-}}$ $A_{R}$, the differential absorbance for left and right circularly polarized light. In a simple two-state model, the pump induced CD changes can be written as follows:

$$
\Delta \mathrm{CD}=\left(\mathrm{CD}_{\mathrm{ES}}-\mathrm{CD}_{\mathrm{GS}}\right) \times \mathrm{N}_{\mathrm{ES}}
$$

where $\mathrm{N}_{\mathrm{ES}}$ is the fraction of excited state population and $\mathrm{CD}_{\mathrm{ES}}$ and $\mathrm{CD}_{\mathrm{GS}}$ are the $\mathrm{CD}$ signals associated to the ground state and the excited state, respectively. Measurements performed with several sample cells and different exposure times to the UV pump revealed the presence of small long-lived signals of a few mdeg in the TR-CD signals. These small signals whose the amplitude and the sign were found to depend on those parameters are attributed to birefringence stemming from the quartz cell windows. In order to increase the signal to noise ratio of the TR-CD signals, the sample cell was kept fixed during the measurements to limit the contribution of the birefringence artefacts to ca. $\pm 5 \mathrm{mdeg}(i$. . $\left.1,5 \times 10^{-4} \mathrm{OD}\right)$. To avoid photodegradation, $15-\mathrm{ml}$ sample solutions were recirculated.

The measured kinetics traces were fitted to a sum of exponential functions and a step function convoluted with a Gaussian function representing the instrumental response function (IRF). The step function accounts for the birefringence artefacts. The presence of two-photon absorption (TPA) peak from the solvent when both pump and probe overlap in time was taken into account by adding to the fitting function a weighted IRF Gaussian. The full width at half maximum (FWHM) of the Gaussian was found to be $\sim 0.9 \mathrm{ps}$ at the probed wavelengths. As an example, figure $\mathrm{S} 1$ illustrates one and two-exponential fits with a step function of the kinetic traces of (Ra)-PL1 measured in cyclohexane. 


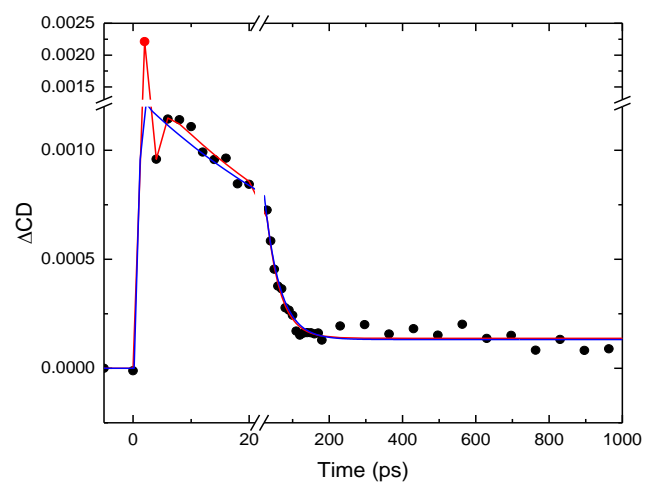

Figure S1: One- and two-exponential fits avec a step function of theTR-CD changes in OD recorded for $\left(\mathrm{R}_{\mathrm{a}}\right)$-PL1 in cyclohexan e. Excitation: $266 \mathrm{~nm}$.

\section{Quantum chemical calculations}

\subsection{Computational details}

Density Functional Theory (DFT) was used to optimize the ground state of $\left(S_{a}\right)$-binol, $\left(R_{a}\right)$-binol, $\left(R_{a}\right)-P L 1$ and $\left(R_{a}\right)$ PL2. Their static CD and absorption spectra as well as their excited Potential Energy Surfaces (PES) were characterized by means of the time dependent version of DFT (TD-DFT) by using the CAM-B3LYP functional. ${ }^{3}$ We also used the M052 $\mathrm{X}^{4,5}$ functional. Both functionals provided a similar picture, indicating that our conclusions do not depend on the choice of the functional. Unless otherwise specified, geometry optimizations were performed by using the 6-31G(d) basis set, whereas the energies were refined by using the larger 6-31+G $(\mathrm{d}, \mathrm{p})$ basis set. Solvent (cyclohexane) effects were included in the calculations implicitly by the Polarizable Continuum Model (PCM) ${ }^{6,7}$ All the calculations were performed using the Gaussian 09. ${ }^{8}$ We also adopted DALTON2016 to compute the excited-state absorption and CD from the four lowest excited states and the transition moments between excited states from the double residues of quadratic response functions. ${ }^{9}$ Due to their computational cost, calculations were only performed in gas-phase with the small 6-31G $(\mathrm{d})$ basis set.

\subsection{Binol conformers}

Ground-state minima ( $\left.S_{0}-\min \right)$ of $\left(S_{a}\right)$-binol, $\left(R_{a}\right)$-binol, $\left(R_{a}\right)$-PL1 and $\left(R_{a}\right)$-PL2 in cyclohexane were optimized with $\mathrm{PCM} / \mathrm{DFT}$. For $\left(\mathrm{S}_{\mathrm{a}}\right)$-binol, we analyzed different orientations of the $\mathrm{OH}$ groups with respect to the aromatic rings (Figure S2). We have then focused our study on the most stable arrangement, in which the $\mathrm{OH}$ groups lie in the molecular plane of its aromatic ring and point towards the opposite ring, denoted double-cis orientation. Note that the energy difference between these conformers in cyclohexane (ca. $>4 \mathrm{kcal} / \mathrm{mol}$ ) exceeds the thermal energy, so that only the double-cis conformer is expected to be present at room temperature, while in protic solvents other conformers could be populated. 

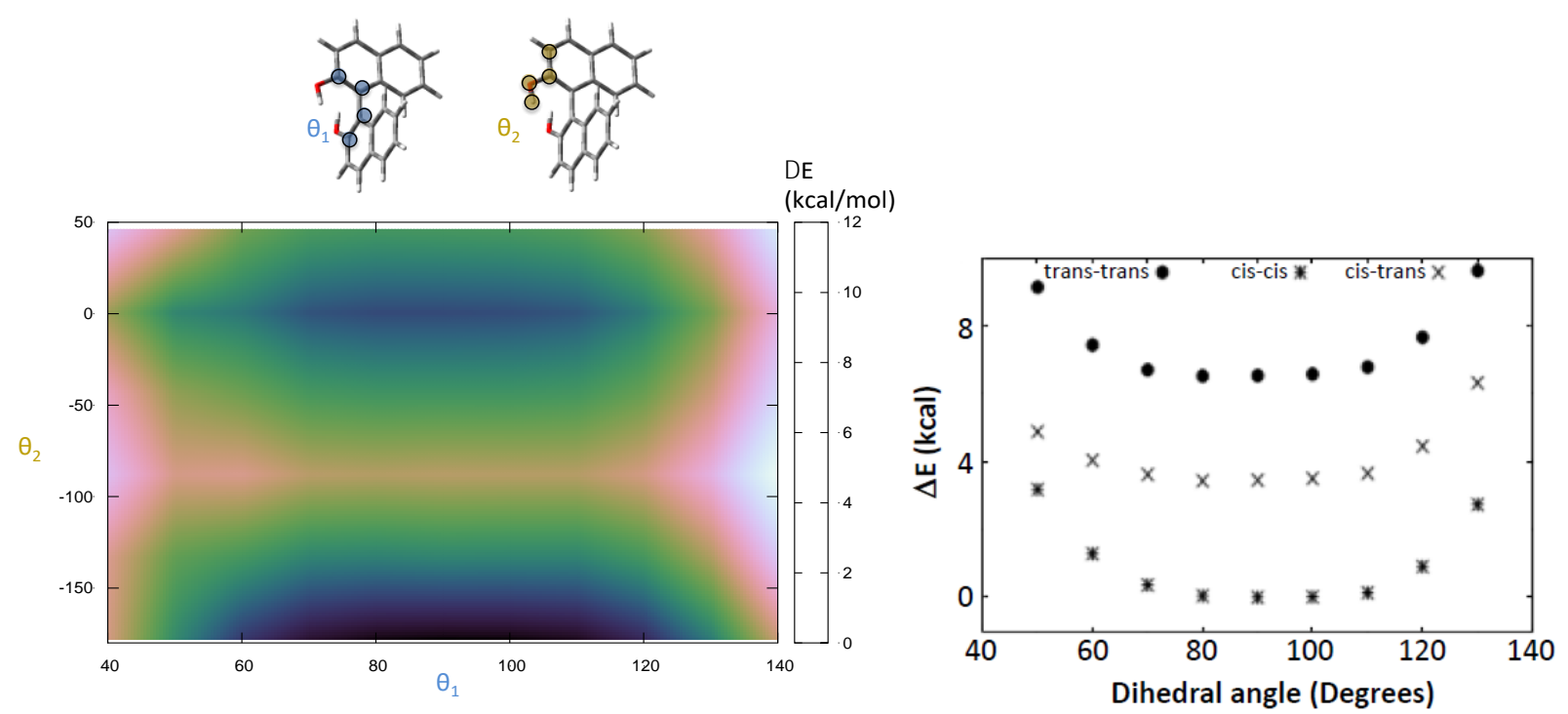

Figure S2. (Left) $2 \mathrm{D}$ variation of PES as a function of $\theta_{1}$ and $\theta_{2}$, in degrees. CAM-B3LYP/6-31G(d). (Right) Torsional potentials for the cis-cis, trans-trans and cis-trans conformers of $\left(S_{a}\right)$-binol as a function of their dihedral angle $\theta$. CAM-B3LYP/6-31G(d) in cyclohexane.

\subsection{Electronic excitations of $\left(S_{a}\right)$-binol, $\left(R_{a}\right)$-PL1, $\left(R_{a}\right)$-PL2 and 2-naphthol}

Table $S 1$ reports the excited-state properties of $\left(S_{a}\right)$-binol and $\left(R_{a}\right)$-PL1 and $\left(R_{a}\right)-P L 2$ calculated at $S_{0}$-min. In agreement with our experimental spectra, the computed absorption spectrum of $\left(\mathrm{S}_{\mathrm{a}}\right)$-binol (in double-cis conformation) exhibits, below $6 \mathrm{eV}$, three main exciton bands with significant oscillator strengths:

- $\mathrm{S}_{0} \rightarrow \mathrm{S}_{1}(4.35 \mathrm{eV})$ transition that mainly results from the anti-symmetric combination of ${ }^{1} \mathrm{~L}_{\mathrm{a}}$ transitions of the 2-naphthol rings corresponding to $\mathrm{HOMO} \rightarrow$ LUMO excitation. $\mathrm{S}_{0} \rightarrow \mathrm{S}_{2}$ is a very weak transition that is the symmetric counterpart of $S_{0} \rightarrow S_{1}$ transition and is $0.06 \mathrm{eV}$ less stable.

- $\mathrm{S}_{0} \rightarrow \mathrm{S}_{3}(4.75 \mathrm{eV})$ transition that stems from the anti-symmetric combination of the ${ }^{1} \mathrm{~L}_{\mathrm{b}}$ transitions of the 2naphthol rings. It corresponds to a mixture of $\mathrm{HOMO}-1 \rightarrow \mathrm{LUMO}$ and $\mathrm{HOMO} \rightarrow \mathrm{LUMO}+1$ excitations. $\mathrm{S}_{0} \rightarrow \mathrm{S}_{4}$ transition is the 'dark' symmetric counterpart very close in energy $(0.02 \mathrm{eV})$.

- $\mathrm{S}_{0} \rightarrow \mathrm{S}_{7}(5.64 \mathrm{eV})$ and $\mathrm{S}_{0} \rightarrow \mathrm{S}_{8}(5.69 \mathrm{eV})$ transitions that are responsible of the most intense absorption band of binol at $226 \mathrm{~nm}$. They arise from the symmetric and anti-symmetric combinations of the ${ }^{1} \mathrm{~B}_{\mathrm{b}}$ transitions of the 2-naphtol rings.

Table S1 : Assignment, vertical absorption energy $(\Delta \mathrm{E}$ in $\mathrm{eV})$ and oscillator strength $(\mathrm{f})$ of the main lowest energy excited

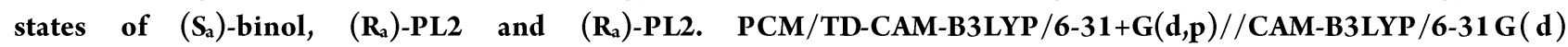
calculations.

\begin{tabular}{|c|c|c|c|c|c|c|}
\hline \multicolumn{7}{|c|}{ Absorption } \\
\hline & \multicolumn{2}{|c|}{$\left(S_{a}\right)$-binol } & \multicolumn{2}{|c|}{$\left(R_{a}\right)-P L 1$} & \multicolumn{2}{|c|}{$\left(R_{a}\right)$-PL2 } \\
\hline \multirow[t]{2}{*}{ State $\backslash \theta$} & \multicolumn{2}{|c|}{$\left(91^{\circ}\right)$} & \multicolumn{2}{|c|}{$\left(-49^{\circ}\right)$} & \multicolumn{2}{|c|}{$\left(-61^{\circ}\right)$} \\
\hline & $\Delta \mathbf{E}$ & $\mathbf{f}$ & $\Delta \mathbf{E}$ & f & $\Delta \mathbf{E}$ & f \\
\hline$S_{1}\left(L_{a}-1\right)$ & 4.35 & 0.20 & 4.21 & 0.41 & 4.35 & 0.36 \\
\hline$S_{2}\left(L_{a}-2\right)$ & 4.41 & 0.02 & ${ }^{*} 4.47$ & 0.01 & *4.50 & 0.00 \\
\hline$S_{3}\left(L_{b}-1\right)$ & 4.75 & 0.13 & ${ }^{*} 4.58$ & 0.01 & *4.60 & 0.02 \\
\hline$S_{4}\left(L_{b}-2\right)$ & 4.77 & 0.00 & 4.63 & 0.00 & 4.68 & 0.00 \\
\hline$S_{5}$ & 4.98 & 0.00 & 4.93 & 0.01 & 4.99 & 0.01 \\
\hline$S_{6}$ & 4.98 & 0.01 & 5.06 & 0.02 & 5.07 & 0.01 \\
\hline $\mathbf{S}_{7}\left(\mathbf{B}_{\mathbf{b}}-\mathbf{1}\right)$ & 5.64 & 1.24 & 5.62 & 1.31 & 5.67 & 1.48 \\
\hline$S_{8}\left(B_{b}-2\right)$ & 5.69 & 0.69 & 5.55 & 0.49 & 5.53 & 0.56 \\
\hline
\end{tabular}

From a quantitative point of view, TD-DFT fails to reproduce the relative ordering of the two bright ${ }^{1} \mathrm{~L}$-derived transitions of $\left(S_{a}\right)$-binol (i.e. $S_{1}$ and $\left.S_{3}\right)$. This is known to arise from the overestimation of the ${ }^{1} L_{a}$ state stability with 
respect to that of the ${ }^{1} \mathrm{~L}_{\mathrm{b}}$ state of 2-naphthol. ${ }^{10-13}$ Table S2 gives the excited-state properties of the 2-naphthol for two different orientations of the $\mathrm{OH}$ group. Our calculations indicate that the two ${ }^{1} \mathrm{~L}$ transitions are strongly vibronically coupled. As shown in Table S2, the simple rotation of the OH group with respect to the aromatic ring is able to change their relative intensity. Importantly, the most striking difference in the absorption spectrum of binol with respect to that of the 2-naphthol is the same amplitude of the two bright ${ }^{1} \mathrm{~L}$ transitions that suggesting the increased coupling of the two ${ }^{1} \mathrm{~L}$ states in binol. A purposely-tailored quantum vibronic study, well outside the scope of the present paper, would be thus necessary to fully assess the interplay between the different excited states of naphthol derivatives.

Table S2. CAM-B3LYP (two different basis sets) in cyclohexane of 2-naphthol (NpOH) exhibiting in plane and perpendicular $O-H$ bonds. Energy in $\mathrm{eV}$, oscillator strength $(f)$ in parentheses.

\begin{tabular}{|c|c|c|c|c|}
\hline 6-31G(d) & \multicolumn{2}{|c|}{ NpOH OH-0 } & \multicolumn{2}{|c|}{ NpOH OH-90 } \\
\hline$\Delta \mathbf{E}$ & \multicolumn{2}{|c|}{0.00} & \multicolumn{2}{|c|}{$3.63 \mathrm{kcal} / \mathrm{mol}$} \\
\hline & Assignment & Energy $(f)$ & Assignment & Energy $(f)$ \\
\hline $\mathrm{S}_{1}$ & $\pi \pi^{*}\left(\mathrm{~L}_{\mathrm{a}}\right)$ & $4.4440(0.0785)$ & $\pi \pi^{*}\left(L_{a}+\mathbf{L}_{\mathbf{b}}\right)$ & $4.6700(0.0407)$ \\
\hline $\mathrm{S}_{2}$ & $\pi \pi^{*}\left(\mathrm{~L}_{\mathrm{b}}\right)$ & $4.8639(0.0309$ & $\pi \pi^{*}\left(\mathbf{L}_{\mathbf{a}}+\mathrm{L}_{\mathrm{b}}\right)$ & $4.8346(0.0630)$ \\
\hline $\mathrm{S}_{3}$ & $\pi \pi^{*}\left(\mathrm{~B}_{\mathrm{b}}\right)$ & $5.9505(1.4646)$ & $\pi \pi^{*}\left(\mathrm{~B}_{\mathrm{b}}\right)$ & $6.0532(1.5227)$ \\
\hline \multicolumn{5}{|l|}{ 6-31+G(d,p) } \\
\hline \multirow[t]{2}{*}{$\Delta \mathbf{E}$} & \multicolumn{2}{|c|}{0.00} & \multicolumn{2}{|c|}{$3.06 \mathrm{kcal} / \mathrm{mol}$} \\
\hline & Assignment & Energy $(f)$ & Assignment & Energy (f) \\
\hline$S_{1}$ & $\pi \pi^{*}\left(\mathrm{~L}_{\mathrm{a}}\right)$ & $4.3613(0.0801)$ & $\pi \pi^{*}\left(\mathrm{~L}_{\mathrm{a}}+\mathrm{L}_{\mathrm{b}}\right)$ & $4.5860(0.0483)$ \\
\hline$S_{2}$ & $\pi \pi^{*}\left(\mathrm{~L}_{\mathrm{b}}\right)$ & $4.7729(0.0312)$ & $\pi \pi^{*}\left(\mathrm{~L}_{\mathrm{a}}+\mathrm{L}_{\mathrm{b}}\right)$ & $4.7495(0.0613)$ \\
\hline $\mathrm{S}_{3}$ & $\pi \pi^{*}$ & $5.5997(0.0000)$ & $\pi \pi^{*}\left(\mathrm{~B}_{\mathrm{b}}\right)$ & $5.8274(1.4364)$ \\
\hline $\mathrm{S}_{4}$ & $\pi \pi^{*}\left(\mathrm{~B}_{\mathrm{b}}\right)$ & $5.7347 \quad(1.5468)$ & & \\
\hline
\end{tabular}

Though likely overestimating the contribution of $\mathrm{L}_{\mathrm{a}}$-exciton to the two lowest energy bright states of binol, our calculations, nonetheless, provide a fairly good description of the experimental absorption, CD and fluorescence spectra. In order to bring solid interpretations with respect to any overestimation of the former kind of transitions by TD-DFT in 2-naphthol, we mapped the PES of the four lowest energy excited state derived from the ${ }^{1} \mathrm{~L}$ states.

The presence of the bridges in $\left(\mathrm{R}_{\mathrm{a}}\right)$-PL1 and $\left(\mathrm{R}_{\mathrm{a}}\right)$-PL2 induces a significant distortion of the aromatic rings leading to further coupling between the two ${ }^{1} \mathrm{~L}$ transitions. Consequently, our calculations predicted a less resolved absorption spectrum in these compounds, the two lowest energy absorption bands merging together, in agreement with our experimental observations.

\subsection{Electronic excitations of $\left(S_{a}\right)$-binol obtained by using the M11 functional}

Table S3 gathers electronic excitations of $\left(S_{a}\right)$-binol calculated by using M11 functional. The M11 functional predicts that the two most intense low-energy excited states (corresponding to $S_{1}$ and $S_{3}$ ) have similar intensity and that the lowest energy one is slightly less intense, i.e. in better agreement with the experimental spectra than the CAMB3LYP picture. According to $M 11$, the $\mathrm{L}_{a}$ and $\mathrm{L}_{b}$ excitons are strongly mixed in the $\mathrm{FC}$ region.

Table S3. Assignment, vertical absorption energy $(\Delta \mathrm{E}$ in $\mathrm{eV})$ and oscillator strength $(\mathrm{f})$ of the lowest bright energy excited states of $\left(S_{a}\right)$-binol. TD-M1 1/6-31G(d)//CAM-B3LYP/6-31G(d) calculations in the gas phase.

\begin{tabular}{c|c|c}
\hline State & $\Delta \mathrm{E}$ & $\mathrm{f}$ \\
\hline $\mathbf{S}_{\mathbf{1}}\left(\mathbf{L}_{\mathrm{b}}-\mathbf{1}\right)^{\mathbf{a}}$ & 4.58 & 0.13 \\
\hline $\mathbf{S}_{\mathbf{2}}\left(\mathbf{L}_{\mathbf{b}}-\mathbf{2}\right)$ & 4.62 & 0.02 \\
\hline $\mathbf{S}_{\mathbf{3}}\left(\mathbf{L}_{\mathbf{a}}-\mathbf{1}\right)$ & 5.05 & 0.15 \\
\hline $\mathbf{S}_{4}\left(\mathbf{L}_{\mathbf{a}}-\mathbf{2}\right)$ & 5.07 & 0.00 \\
\hline $\mathbf{S}_{7}\left(\mathbf{B}_{\mathbf{b}}-\mathbf{1}\right)$ & 6.09 & 1.28 \\
\hline $\mathbf{S}_{\mathbf{8}}\left(\mathbf{B}_{\mathbf{b}}-\mathbf{2}\right)$ & 6.18 & 0.91 \\
\hline
\end{tabular}

Notes: a) all the four lowest energy excited states are strongly mixed. 


\subsection{Detailed analysis of the torsional potential energy surfaces}

While $S_{2}$ is predicted to keep an orthogonal arrangement of the rings, a partial planarization stabilizes the two lowest bright excited states $\left(S_{1}\right.$ and $\left.S_{3}\right)$. The PESs of both states exhibit two stationary points in C2 symmetry that correspond to a cisoid and transoid conformations illustrated in Figure S3. Frequency analysis confirms that these points correspond to true minima on the PES of $S_{1}$. Therefore, the stable geometry reached after excitation depends on the initial arrangement of the two naphthols rings in $\mathrm{S}_{0}$. Starting from a slightly cisoid conformation $\left(\theta=80^{\circ}\right)$, a cisoid minimum $\left(\mathrm{S}_{1}-\min ^{\text {cis }}\right.$ with $\left.\theta=55.4^{\circ}\right)$ is reached, while starting from the Franck Condon $(\mathrm{FC})$ region $\left(\theta \sim 90^{\circ}\right)$ or from a slightly transoid conformation $\left(\theta=100^{\circ}\right)$, a transoid minimum $\left(\mathrm{S}_{1}-\mathrm{min}^{\text {trans }}\right.$ with $\left.\theta=122.0^{\circ}\right)$ is reached. $\mathrm{S}_{1}$-min ${ }^{\text {trans }}$ is the absolute minimum, being $c$ a. $0.33 \mathrm{eV}(0.19 \mathrm{eV}$ with the larger 6-31+G $(\mathrm{d}, \mathrm{p})$ basis set $)$ more stable than $\mathrm{S}_{1}-\mathrm{min}^{\text {cis, }}$, in cyclohexane. The vertical emission energy (VEE) from $\mathrm{S}_{1}-\mathrm{min}^{\text {trans }}$ is $3.43 \mathrm{eV}$ (oscillator strength, $\mathrm{f}=0.39$ ) and $3.66 \mathrm{eV}$ $(\mathrm{f}=0.37)$ from $\mathrm{S}_{1}-\mathrm{min}^{\mathrm{cis}}$, these values being consistent with the observed broad experimental fluorescence spectrum of binol. Regarding $S_{3}$, the frequency analysis shows that both $S_{3}-m^{\text {in }}{ }^{\text {trans }}$ and $S_{3}-\mathrm{min}^{\text {cis }}$ exhibit an imaginary frequency along a nontotal-symmetric (B) ring stretching mode, indicating that these structures do not correspond to absolute minima of the PES. Restarting the geometry optimization after a distortion along the imaginary mode leads to two true minima in the $S_{3}$ PES, $S_{3}$-min-loc ${ }^{\text {trans }}$ and $S_{3}-$ min $-\operatorname{loc}^{\text {cis }}$ where the excitation is mainly localized on one of the rings as shown in Figure $S 4$. These two minima are slightly more stable $(\sim 0.5-0.6 \mathrm{kcal} / \mathrm{mol})$ than their delocalized counterpart.

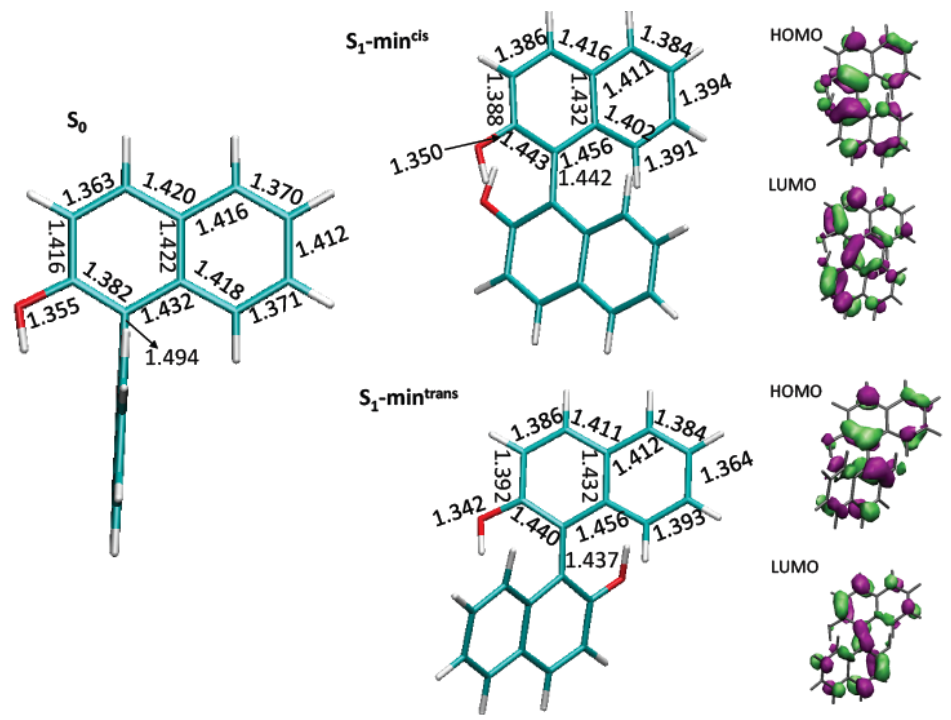

Figure S3. Main geometric parameters of the ground and $S_{1}$ minima for $\left(S_{a}\right)$-binol in cyclohexane as predicted by PCM/CAMB3LYP/6-31G(d) calculations. The frontier orbitals in the excited state minima are also shown.

Our calculations predict that the $\mathrm{L}_{\mathrm{a}}-1$ state $\left(\mathrm{S}_{1}\right)$ is more sensitive to the dihedral angle than the other excited states, since it is characterized by the largest planarization $\left(\Delta \theta=40-50^{\circ}\right)$ and by the largest energy stabilization, i.e. $\sim 13 \mathrm{kcal} / \mathrm{mol}$ with respect to the FC point. Figure S3 shows that the LUMO has a significant bonding character with respect to the $\mathrm{C} 2-\mathrm{C} 2$ ' atoms, explaining why $S_{1}$ is stabilized by the binol planarization, which also increases the coupling between the two aromatic rings. The strong stabilization of $S_{1}$ after the geometry relaxation suggests that, independently of the wrong en ergy order of the ${ }^{1} \mathrm{~L}$ states predicted by TD-DFT at the FC point, emission from $\mathrm{S}_{1}$-min, is possible. Interestingly, the oscillator strength of $S_{1}$ increases significantly during the geometry optimizations, whereas the opposite is found for $S_{3}$. It is thus clear that the planarization of the rings leads to an increase of the vibronic coupling between the $\mathrm{L}_{\mathrm{a}}$-derived transitions. On the other hand, for the $\mathrm{L}_{\mathrm{b}}$-derived transitions, which are characterized by a smaller exciton coupling, geometry optimizations can lead to a partial localization of the excitation, this feature being also modulated by the dihedral angle. Emission for $S_{3}$-min-loc is predicted to be weak ( $f \sim 0.02$ ) falling at $4.44 \mathrm{eV}$ (for $\mathrm{S}_{3}$-min-loc ${ }^{\text {trans }}$ ) or $4.47 \mathrm{eV}$ (for $\mathrm{S}_{3}$-min-loc ${ }^{\text {cis }}$ ) with a very small Stokes shift with respect to the absorption maximum. Delocalized C2-symmetric pseudo-minima emit slightly on the blue ( 4.48 for $\mathrm{S}_{3}$-min-loc ${ }^{\text {trans }}$ and $4.54 \mathrm{~S}_{3}$-min-loc $\left.{ }^{\text {cis }}\right)$ with a larger intensity $(0.03 \sim 0.05)$. On the balance, our calculations suggest that the $\mathrm{L}_{b}$-like minima contribute to the bluewing of the experimental fluorescence spectrum. 


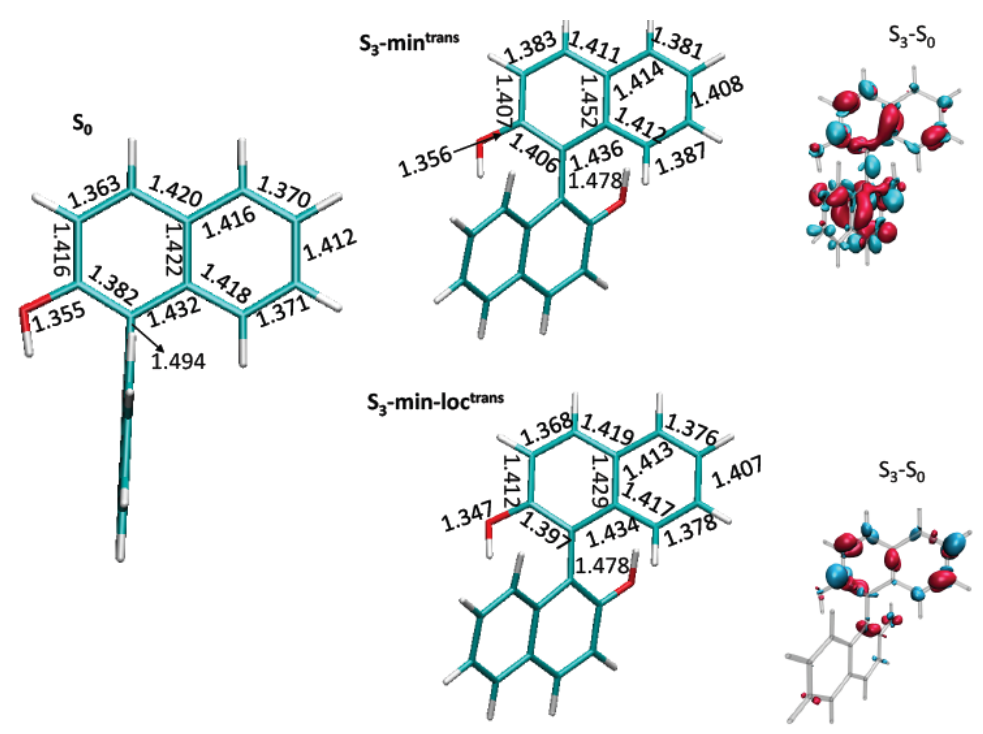

Figure S4. Main geometric parameters of the ground state and $S_{3}$ minima (delocalized and partially localized) for $\left(S_{a}\right)$-binol in cyclohexane as predicted by PCM/CAMB3LYP/6-31G(d) calculations. The electronic density difference associated to the electronic transition in the excited state minima is also shown.

Regarding $\left(\mathrm{R}_{\mathrm{a}}\right)$-PL1 and $\left(\mathrm{R}_{\mathrm{a}}\right)$-PL2, optimizations of their $\mathrm{S}_{1}$ geometry also indicate a decrease of $\theta$. At the minimum, the rings rotate towards a slightly more-cisoid conformation. On the other hand, since their two rings already adopt a cisoid conformation in $\mathrm{S}_{0}$, their degree of rotation is significantly lower than that of $\left(\mathrm{S}_{\mathrm{a}}\right)$-binol, i.e. $-4^{\circ}$ for $\left(\mathrm{R}_{\mathrm{a}}\right)-\mathrm{PL1}$ and $-14^{\circ}$ for $\left(R_{a}\right)$-PL2 as shown in Figure $S 5$. A small increase of $\theta$ is predicted for both bridged compounds in $S_{2}$ and $S_{3}: 1^{\circ}$ for $\left(R_{a}\right)$-PL1 and $\sim 4^{\circ}$ for $\left(R_{a}\right)$-PL2, respectively. Unlike $\left(S_{a}\right)$-binol, the fully delocalized excited structures are found to be real minima of the $S_{3}$ PES. In fact, we obtain a qualitative picture similar to that of $\left(S_{a}\right)$-binol, considering that both $\left(\mathrm{R}_{\mathrm{a}}\right)$-PL1 and $\left(\mathrm{R}_{\mathrm{a}}\right)-\mathrm{PL} 2$ are already in a constrained cisoid conformation in the ground state. The computed emission energy from $S_{1}$-min is $3.44 \mathrm{eV} \mathrm{eV}$ for $\left(\mathrm{R}_{\mathrm{a}}\right)$-PL1 and $3.57 \mathrm{eV}$ for $\left(\mathrm{R}_{\mathrm{a}}\right)$-PL2, nicely reproducing the blue-shift of the latter compound found in the experimental fluorescence spectra. Also for these compounds, emission from $\mathrm{S}_{3}$-min could contribute to the blue-wing of the experimental spectra.

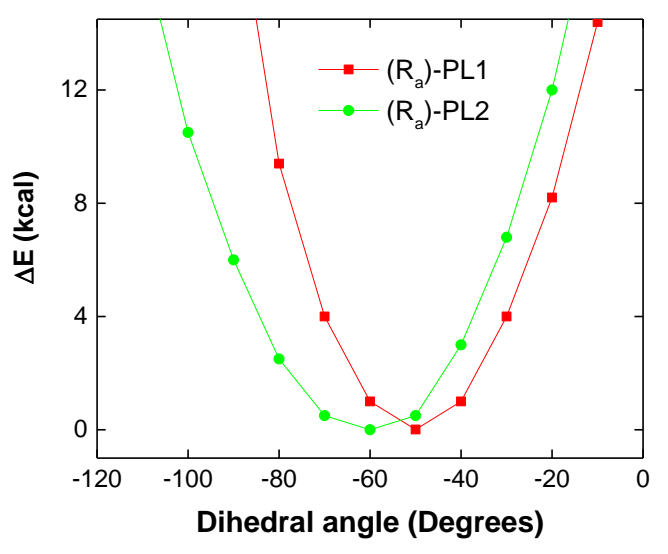

Figure S5. Torsional potentials for $\left(R_{a}\right)-P L 1$ and $\left(R_{a}\right)-P L 2$ in $S_{0}$ as a function of their dihedral angle $\theta$. CAM-B3LYP/6-31G(d) in cyclohexane.

It is worth noting that our geometry optimizations of $S_{1}$ obtained with PCM/TD-M11/6-31G(d) that correctly predict the ordering of the ${ }^{1} \mathrm{~L}$ states also leads to a $\mathrm{L}_{\mathrm{a}}$-like exciton minimum, with emission energy of $3.64 \mathrm{eV}$, characterized by a significant planarization $\left(\theta=124^{\circ}\right)$ and a high oscillator strength $(\mathrm{f}=0.42)$. Geometry optimization of $\mathrm{S}_{3}$ leads instead to a $\mathrm{L}_{b}$-like minimum with a small planarization $\left(\theta=60^{\circ}\right)$ and a weak oscillator strength $(\mathrm{f}=0.09)$.

\subsection{Computed static CD spectra of binols at the Boltzman equilibrium}




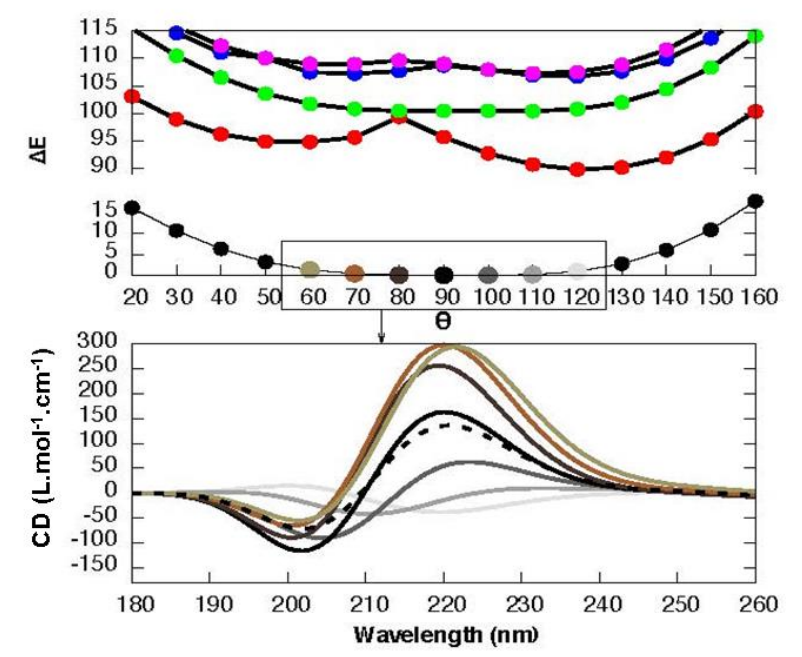

Figure S6. Upper side: relative energy (in kcal) of the five lowest electronic states of $\left(\mathrm{S}_{\mathrm{a}}\right)$-binol for different values of the dihedral angle in degrees $(\theta)$, computed at the PCM/TD-CAM-B3LYP/6-31G(d) level of theory: $S_{0}$ PES in black, $S_{1}$ (bright state) in red, $S_{2}$ (dark state) in green, $S_{3}$ (bright state) in blue, $S 4$ (dark state) in pink. Down side: calculated CD spectra of $\left(S_{a}\right)$-binol as a function of the dihedral angle $(\theta)$. CAM-B3LYP/6-31G(d) in cyclohexane.

\section{Steady-state fluorescence in ethanol and ethylene glycol}
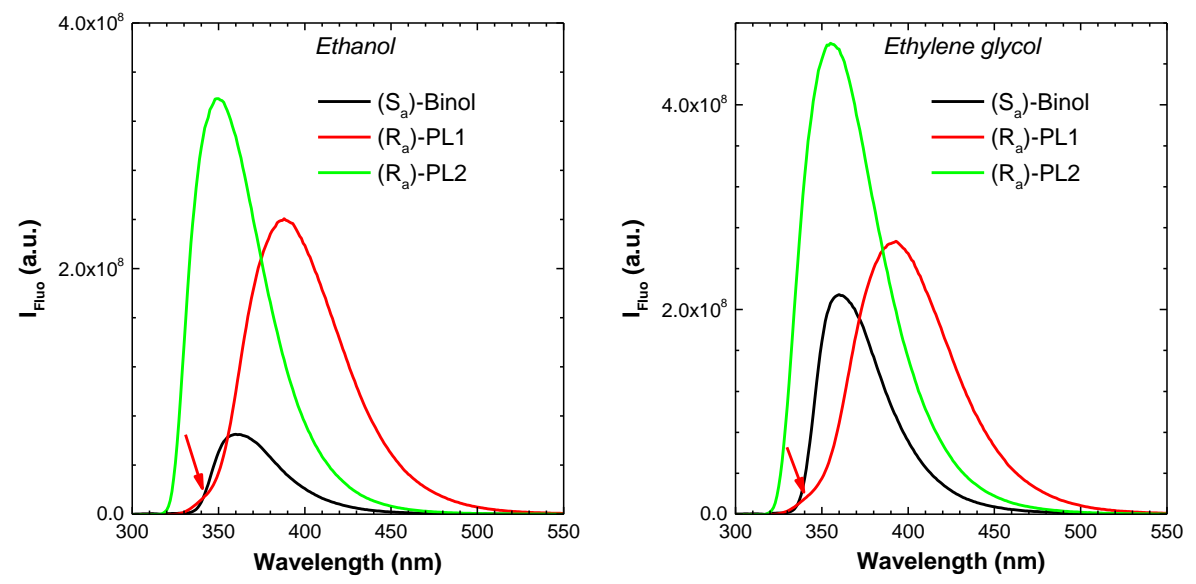

Figure S7. Steady-state fluorescence spectra of $\left(S_{a}\right)$-binol, $\left(R_{a}\right)$-PL1 and $\left(R_{a}\right)$-PL2 in ethanol and ethylene glycol. The relative spectral areas are representative of the fluorescence quantum yields. Red arrows indicate the small shoulder in the fluorescence of ( $\left.\mathrm{R}_{\mathrm{a}}\right)$-PL1. Excitation wavelength: $\lambda=290 \mathrm{~nm}$.

Table S4. Solvent parameters.

\begin{tabular}{cccc}
\hline Solvents & $\eta(\mathbf{m P a} . \mathbf{s})$ & $\boldsymbol{\alpha}$ & $\boldsymbol{\beta}$ \\
\hline Cyclohexane & $0.98\left(20^{\circ} \mathrm{C}\right)$ & 0 & 0 \\
Ethanol & $1.20\left(20^{\circ} \mathrm{C}\right)$ & 0.86 & 0.75 \\
Ethylene Glycol & $16.06\left(25^{\circ} \mathrm{C}\right)$ & 0.9 & 0.52 \\
\hline
\end{tabular}

$\eta, \alpha$ and $\beta$ are respectively the viscosity, the hydrogen-bond donor and acceptor characters of the solvent taken from ref 14 . 


\section{Additional time-resolved absorption and CD measurements}

\subsection{Test for the validity of the TR-CD set-up}

As time-resolved ellipticity measurements are prone to polarization artifacts, we performed a preliminarystudy of the two binol enantiomers in ethanol, in order to validate our experimental set-up. Achiral $\Delta \mathrm{A}$ signals and $\Delta \mathrm{CD}$ kinetic traces were averaged over 300 shots. As shown in Figure S8, both $\Delta \mathrm{A}$ and $\Delta \mathrm{CD}$ signals exhibit a strong peak around the time zero stemming from two photon absorption (TPA) by the solvent (Figure S9).

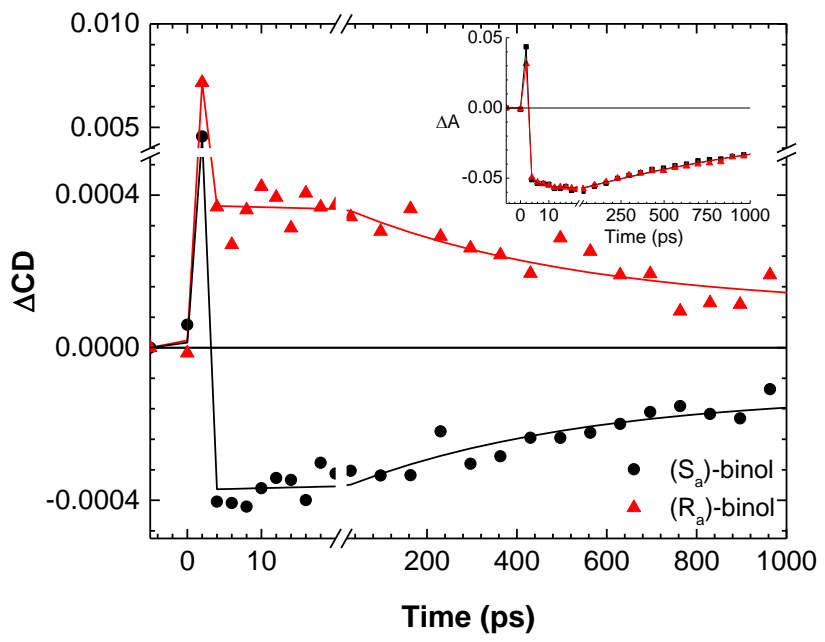

Figure S8: TR-CD and transient absorption changes in OD (insert) at $234 \mathrm{~nm}$ recorded for $\left(\mathrm{R}_{\mathrm{a}}\right)$ - and $\left(\mathrm{S}_{\mathrm{a}}\right)$-binols in ethanol. Excitation: $266 \mathrm{~nm}$.

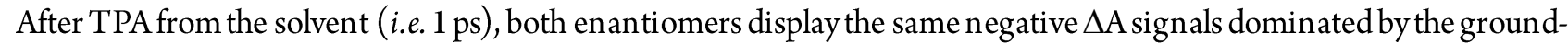
state bleaching. The $\triangle \mathrm{A}$ kinetic traces were globally fitted with the sum of the IRF Gaussian function and a two exponential function convoluted to the IRF Gaussian function. The fit yields one delayed rising component of $4.9 \pm$ $0.7 \mathrm{ps}$ and a decay of $1.76 \pm 0.02 \mathrm{~ns}$. The nanosecond time component is ascribed to the excited state lifetime. In contrast, the short delayed rising component of the bleaching signal may be due to the contribution of a fast spectral shift of the excited-state absorption (ESA) band, known to contribute in this spectral region. As expected, after $1 \mathrm{ps,}$ the $\triangle \mathrm{CD}$ signals associated to the two enantiomers behave similarly but with opposite sign. They reflect the $\mathrm{CD}$ changes induced by the excitation corresponding to the ground-state bleaching due to the dominant contribution of $\mathrm{CD}_{\mathrm{Gs}} \Delta \mathrm{CD}$ signals of the two enantiomers were globally fitted with an exponential function with the time components of $590 \pm 50 \mathrm{ps}$ and a step function arising from the contribution of the cell birefringence. The temporal changes of the $\Delta \mathrm{CD}$ signals are found to be about three times faster than that of $\Delta \mathrm{A}(590 \mathrm{ps} v s .1 .76 \mathrm{~ns})$ indicating that they mainly stem from the temporal variation of $\mathrm{CD}_{\mathrm{ES}}$. The absence of the short lifetime of 18 ps observed in Figure 2 is explained by the lower accuracy of these preliminary measurements that were averaged over 300 shots $v s .1000$ shots.
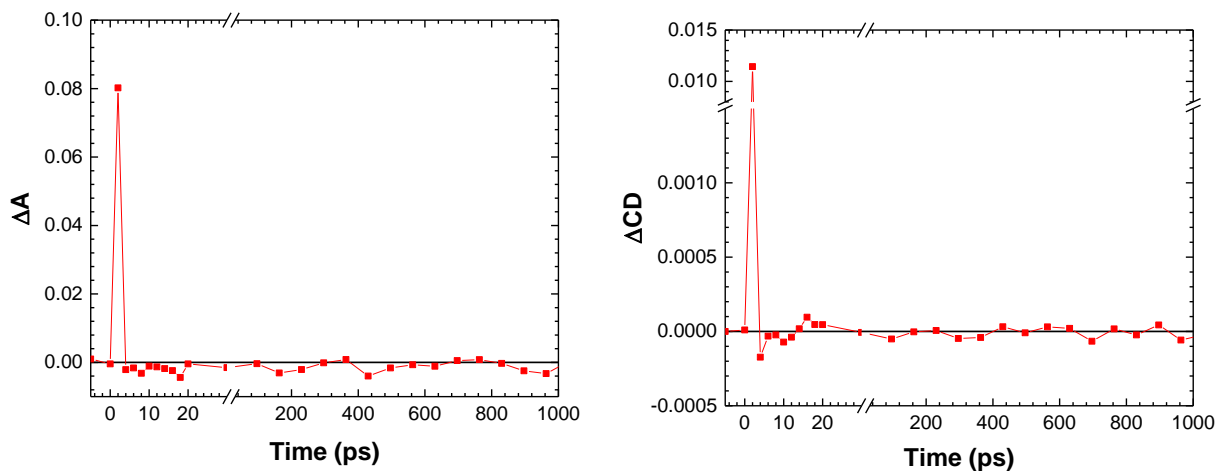

Figure S9. (Left) Transient absorption changes and (Right) TR-CD changes, in OD, measured in ethanol. Excitation and probe wavelengths: $266 \mathrm{~nm}$ and $234 \mathrm{~nm}$.

\subsection{Achiral transient absorption measurements}




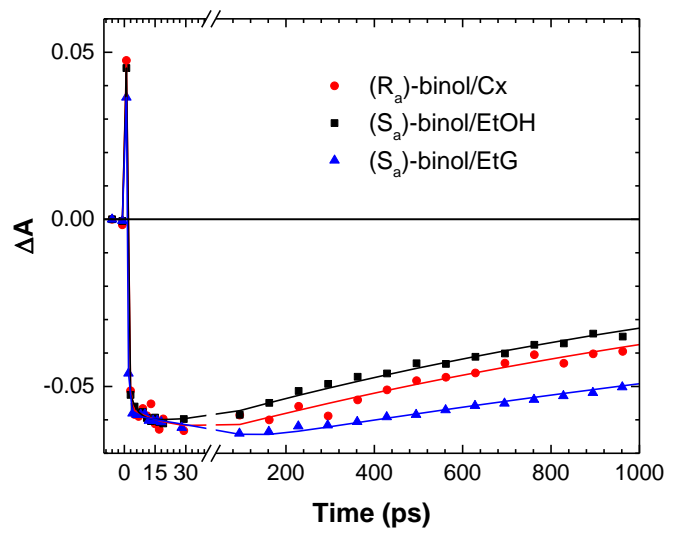

Figure S1 0. Transient absorption changes measured for $\left(S_{a}\right)$-binol in cyclohexane and $\left(R_{a}\right)$-binol in ethanol and ethylene glycol. Excitation and probe wavelengths: $266 \mathrm{~nm}$ and $234 \mathrm{~nm}$.
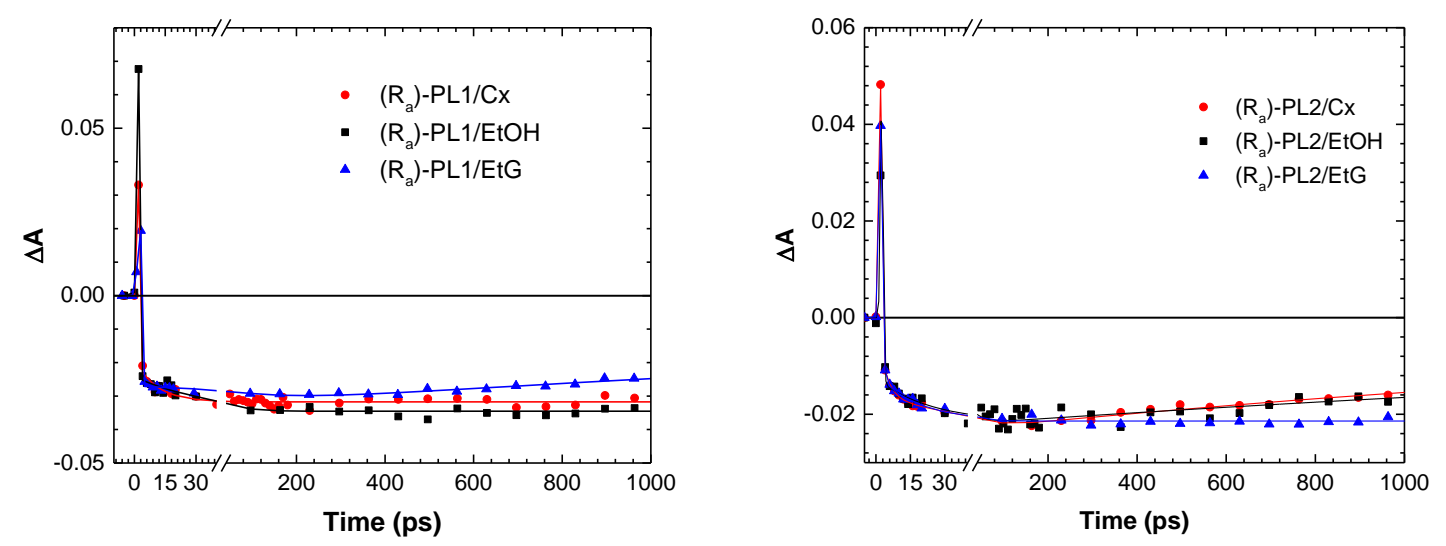

Figure S1 1. Transient absorption changes measured for $\left(R_{a}\right)$-PL1 and ( $\left.R_{a}\right)$-PL2 in cyclohexane, ethanol and ethylene glycol. Excitation and probe wavelengths: $266 \mathrm{~nm}$ and $234 \mathrm{~nm}$.

\section{Excited-state CD calculations}

Excited-state CD calculations were performed with DALTON from the double residues of quadratic response functions in gas-phase. When possible, we exploited symmetry, accounting for the lowest 25 states of both A and B symmetry. For the asymmetric partially-localized $\mathrm{L}_{\mathrm{b}}-1$ minimum, we computed 30 excited states. All spectra fully converged with the number of states up to $3 \mathrm{eV}$. In order to reduce the computational cost, we resorted to the $6-31 \mathrm{G}(\mathrm{d})$ basis set. Previous CAM-B3LYP calculations from the first 4 excited states of $\left(\mathrm{R}_{\mathrm{a}}\right)$-binol to the lowest 8 excited states adopting a large basis set aug-cc-pVTZ but covering only $0.8 \mathrm{eV}$ provided a useful reference for our study. ${ }^{15}$

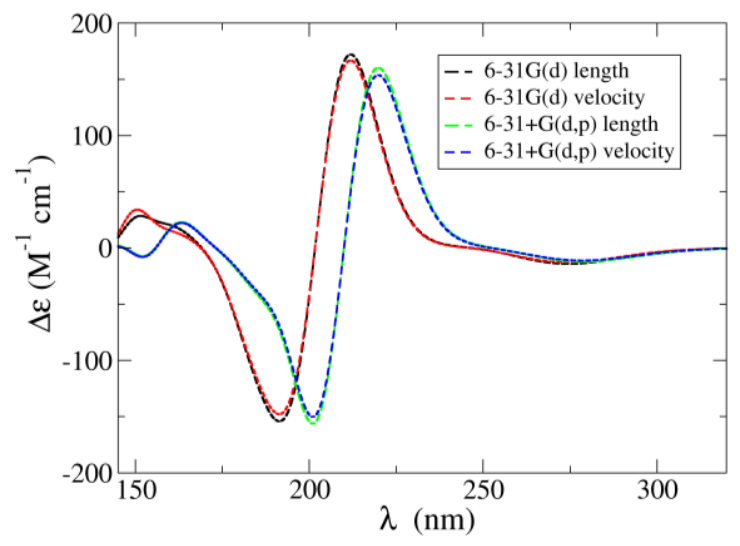

Figure S12. Comparison of the calculated $C_{G}$ spectra of $\left(S_{a}\right)$-binol in gas phase obtained at CAM-B3LYP/6-31G(d) level of theory with length and velocity gauges. 
Since length-gauge calculations may suffer from origin-dependence problems and velocity-gauge calculations may converge slowly with the dimension basis set, we first checked that the computations of the ground-state $\mathrm{CD}\left(\mathrm{CD}_{\mathrm{GS}}\right)$ with velocity and length gauges with 6-31G(d) basis set provide very similar results. Figure S12 shows that, apart from a small blue-shift of $C D_{\mathrm{GS}}$, results of our calculations with the 6-31G(d) basis set are very similar to those obtained with the larger 6-31+G $(\mathrm{d}, \mathrm{p})$ basis set. It has been previously highlighted that, even with the large aug-cc-pVTZ basis set, the excited-state $\mathrm{CD}\left(\mathrm{CD}_{\mathrm{ES}}\right)$ spectra of binol obtained with the velocity and length gauges show some small differences. ${ }^{15}$ Actually, $\mathrm{CD}_{\mathrm{ES}}$ could be more sensitive to the size of the basis set than $\mathrm{CD}_{\mathrm{GS}}$, since higher-energy states are involved and even the description of the initial states of $\mathrm{CD}_{\mathrm{ES}}$ transitions could significantly change with the adopted basis set. This is particularly true for binol, whose four lowest excited states are quite close in energy, so that the extent they mix can depend on the basis set. Figure S13 illustrates $\mathrm{CD}_{\mathrm{ES}}$ spectra performed with the length and velocity gauges for two geometries: those of the $S_{0}$ minimum and the $\mathrm{L}_{\mathrm{a}}-1\left(\mathrm{~S}_{1}\right)$ minimum in the transoid conformation. The two gauges give qualitatively very similar results. From the quantitative point of view, some discrepancies are noticed in the low-energy window that are larger for the bright $\mathrm{L}_{\mathrm{a}}-1$ and $\mathrm{L}_{b}-1$ states. Figure $\mathrm{S} 13$ shows that the choice of the gauge does not qualitatively affect the shape of the $\mathrm{CD}_{\mathrm{ES}}$ spectra, providing evidence it is not critical for the scope of our investigation.
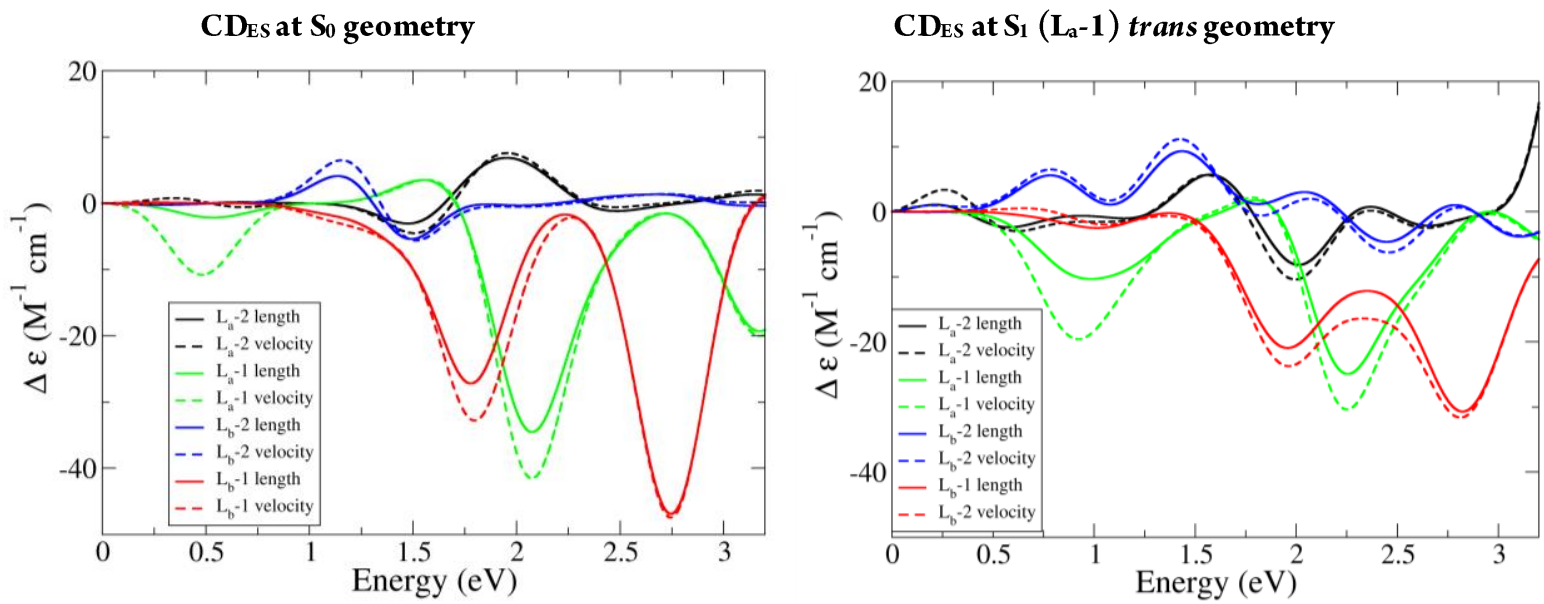

Figure S13. Calculated CDES spectra of the four lowest excited states of $\left(S_{a}\right)$-binol in gas phase obtained at CAM-B3LYP/6$31 \mathrm{G}(\mathrm{d})$ level with length (solid lines) and velocity (dashed lines) gauges, at two different geometries: (left side) the equilibrium geometry of $S_{0}$ and (right side) the transoid minimum of $S_{1}\left(L_{a}-1\right)$. The initial excited state of the transition is indicated in the inset.

Figure $\mathrm{S} 14$ shows the $\mathrm{CD}$ ES spectra computed with the length gauge from the four lowest excited states to higher energy states $\left(S_{n}\right)$ at the different selected geometries. Inspection of the calculated spectra highlights some general trends:

- Each excited state exhibits a different $\mathrm{CD}_{\mathrm{ES}}$ spectrum. However, we notice similarities between the states of same symmetry: $L_{a}-1$ and $L_{b}-1$ (both of symmetry $B$ ) and $L_{a}-2$ and $L_{b}-2$ (both of symmetry $A$ )

- The intensity of the $\mathrm{CD}_{\mathrm{ES}}$ signals is larger for the B-symmetry states than for the A-symmetry states.

- For each state, the $\mathrm{CD}$ ES spectra are significantly shifted on the energy axis at the different geometries, while the change of their shape is moderate. Therefore, considering a single excitation probe, it is predicted that changes in the dihedral angle can result into a change of the intensity of the four excited states.

- At the partially localized transoid geometry of the $\mathrm{L}_{b}-1$ state, the $\mathrm{CD}_{\mathrm{ES}}$ signals of the four excited states exhibit remarkable changes mainly involving significant intensity decreases. 

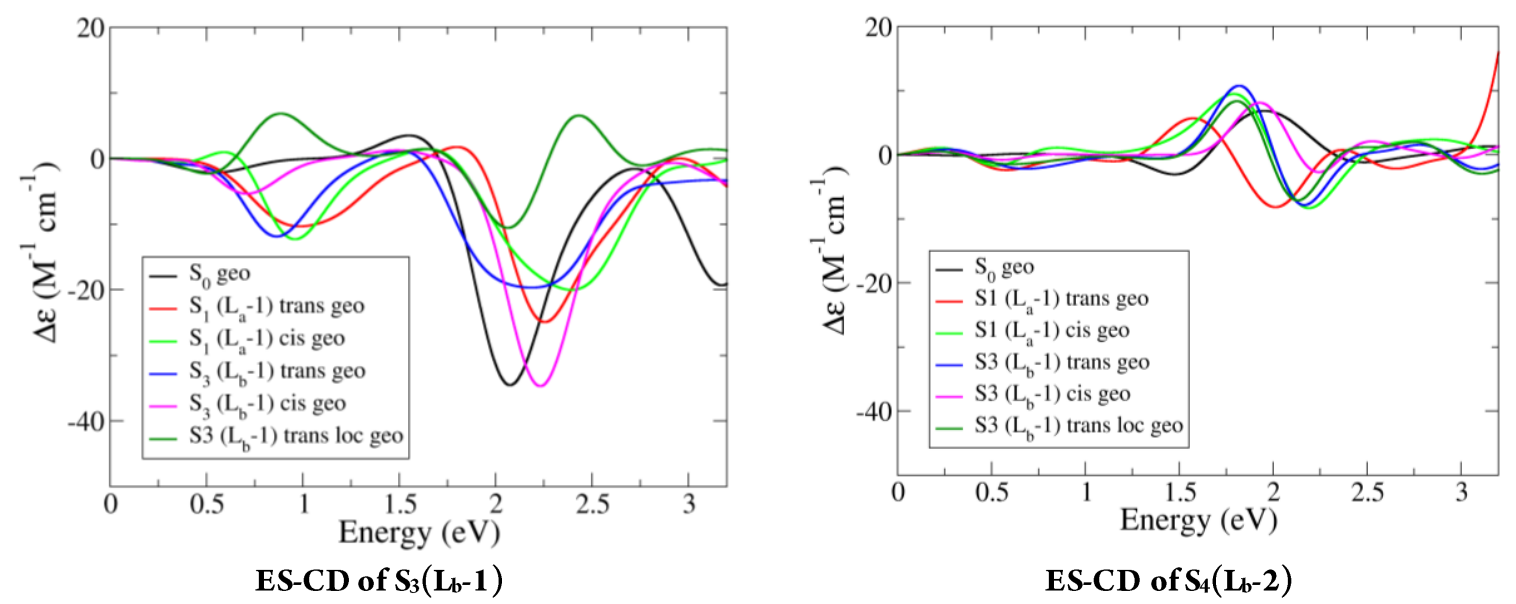

ES-CD of $S_{3}\left(\mathbf{L}_{b}-1\right)$
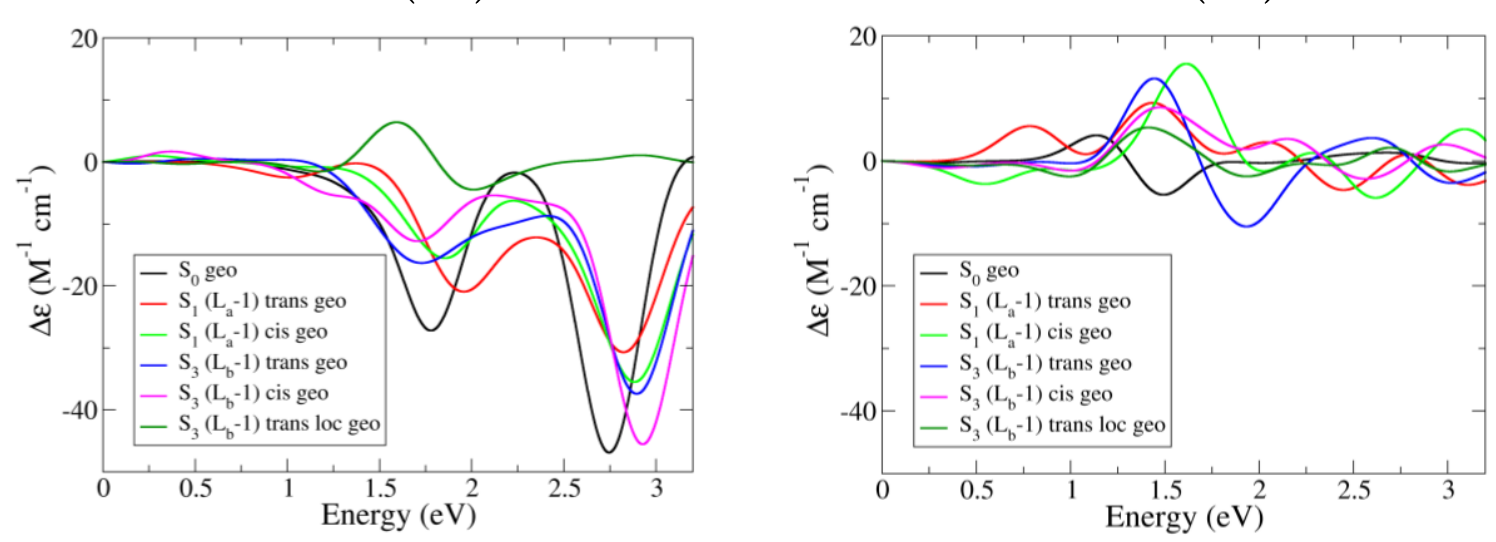

Figure S14. CDES spectra of the four lowest excited states (S1-S4) of $\left(S_{a}\right)$-binol computed at CAM-B3LYP/6-31G(d) level in gas phase at the different geometries indicated in insets.

\section{References}

(1) Simpson, J.E.; Daub, G.H.; Hayes, F.N., The Synthesis of Some 2,2'-Dioxa-Bridged Biphenyls and 1,1'-Binaphthyls, J. Org. Chem. 1973, 38, 1771.

(2) Niezborala, C.; Hache, F., Measuring the dynamics of circular dichroism in a pump-probe experiment with a Babinet-Soleil compensator, J. Opt. Soc. Am. B 2006, 23, 2418-2424.

(3) Yanai, T.; Tew, D.P.; Handy, N.C., A new hybrid exchange-correlation functional using the Coulomb-attenuating method (CAM-B3LYP), Chem. Phys. Lett. 2004, 393, 51-57.

(4) Zhao, Y.; Schultz, N.E.; Truhlar, D.G., Design of Density Functionals by Combining the Method of Constraint Satisfaction with Parametrization for Thermochemistry, Thermochemical Kinetics, and Noncovalent Interactions, J. Chem. Theory Comput. 2006, 2, 364-382.

(5) Zhao, Y.; Truhlar, D.G., Density Functionals with Broad Applicability in Chemistry, Acc. Chem. Res. 2008, 41, 157167.

(6) Miertuš, S.; Scrocco, E.; Tomasi, J., Electrostatic interaction of a solute with a continuum. A direct utilizaion of AB initio molecular potentials for the prevision of solvent effects, Chem. Phys. 1981, 55, 117-129.

(7) Tomasi, J.; Mennucci, B.; Cammi, R., Quantum Mechanical Continuum Solvation Models, Chem. Rev. 2005, 105, 2999-3094.

(8) Frisch, M.J.; Trucks, G. W.; Schlegel, H. B.; Scuseria, G. E. ; et al, Gaussian 09, Revision A.1, in, Gaussian, Inc, Wallingford CT 2009.

(9) Aidas, K.; Angeli, C.; Bak, K.L.; Bakken, V.; et al., The Dalton quantum chemistry program system, Wiley Interdiscip. Rev. Comput. Mol. Sci. 2014, 4, 269-284.

(10) Nishinoto, K., Electronic spectra and structure of a- and b-naphthol, J.Phys. Chem. 1963, 67, 1443-1446. 
(11) Xiao, D.; Prémont-Schwarz, M.; Nibbering, E.T.J.; Batista, V.S., Ultrafast Vibrational Frequency Shifts Induced by Electronic Excitations: Naphthols in Low Dielectric Media,J. Phys. Chem. A 2012, 116, 2775-2790.

(12) Parac, M.; Grimme, S., A TDDFT study of the lowest excitation energies of polycyclic aromatic hydrocarbons, Chem.Phys. 2003, 292, 11-21.

(13) Acharya, A.; Chaudhuri, S.; Bastista, V.S., Can TDDFT describe excited electronic states of naphthol photoacid? A closer look with EOM-CCSD, J. Chem. Theory Comput. 2018, 14, 867-876.

(14) Marcus, Y., The Properties of Organic Liquids that are Relevant to their Use as Solvating Solvents, Chem. Soc. Rev. 1993, 22, 409-416.

(15) Rizzo, A.; Vahtras, O., Ab initio study of excited state electronic circular dichroism. Two prototype cases: Methyl oxirane and R-(+)-1,1'-bi(2-naphthol), J. Chem.Phys. 201 1, 134, 244109. 